\title{
Erratum
}

\section{Erratum to: The treatment effect of novel hGHRH homodimer to male infertility hamster}

\author{
Xu-Dong Zhang, ${ }^{2, \#}$, Xiao-Yuan Guo ",", Jing-Xuan Tang ${ }^{3}$, Lin-Na Yue ${ }^{1}$, Juan-Hui Zhang ${ }^{4}$, Tao Liu ${ }^{6}$, Yu-Xia Dong ${ }^{6}$, and \\ Song-Shan Tang ${ }^{1, *,+}$
}

${ }^{1}$ Department of Biochemistry and Molecular Biology, School of Basic Courses, Guangdong Pharmaceutical University, Guangzhou 510006, ${ }^{2}$ Clinical Laboratories, Guangdong Provincial Corps Hospital of Chinese People's Armed Police Forces, Guangzhou Medical University, Guangzhou 510507, China, ${ }^{3}$ Department of Chemical \& Biological Engineering, School of Engineering, University of Wisconsin-Madison, Madison 53706, USA, ${ }^{4}$ Department of Obstetrics \& Gynecology, Guangdong Provincial Corps Hospital of Chinese People's Armed Police Forces, Guangzhou Medical University, Guangzhou 510507, ${ }^{5}$ Guangdong Provincial Key Laboratory of Pharmaceutical Bioactive Substances, School of Basic Courses, Guangdong Pharmaceutical University, Guangzhou 510006, ${ }^{6}$ Department of Endocrinology, Wuwei City Hospital, Wuwei 733000, China

\section{Erratum to:}

Korean J Physiol Pharmacol 2018;22(6):637-647

https://doi.org/10.4196/kjpp.2018.22.6.637

The authors note that on page 637 (Author Name), author affiliation of Tao Liu "Tao Liu" should instead appear as "Tao Liu." 